\title{
Evaluación de la efectividad de programas de visitas domiciliarias para madres adolescentes y sus hijos/as
}

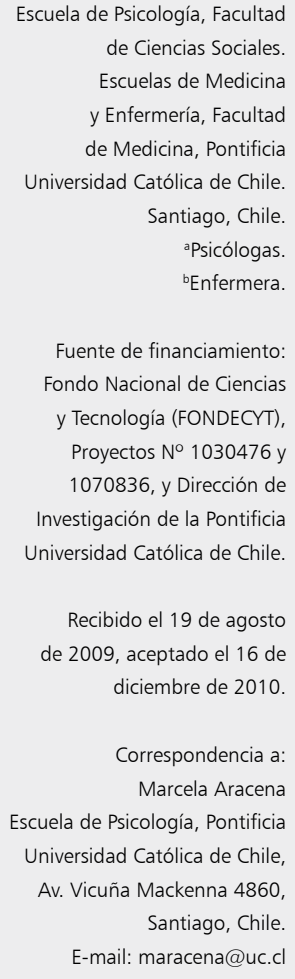

\author{
MARCELA ARACENA ${ }^{a}$, LORETO LEIVAa, CONSUELO UNDURRAGA ${ }^{a}$, \\ MARIANE KRAUSE, CAROLA PÉREZ, VICTORIA CUADRA, \\ MARÍA SILVIA CAMPOS ${ }^{b}$, PAULA BEDREGAL

\section{Effectiveness of a home visit program} \\ for adolescent mothers and their children
}

Background: Home visiting is effective for the promotion and prevention of mother-child health in other countries, especially in vulnerable populations such as pregnant teenagers. Aim: To evaluate the association between receiving a home visiting program during pregnancy and child development during the first year of life, maternal mental health, perception of social support and school attendance. Material and Methods: Cross sectional assessment of 132 teenage mother-sibling pairs. Of these, 87 received home visits and 45 were randomly assigned to a control group. The assessed variables were maternal mental health, perception of social support, life satisfaction, incorporation of mothers to school after delivery, child development and frequency of child abuse and neglect. Results: Mothers that received home visits had a better mental health and went back to school in a higher proportion. No significant differences between groups were observed on perception of social support or child development. Conclusions: These results suggest the effectiveness of domiciliary visits performed by non-professionals, to improve mental health and social integration of teenage mothers.

(Rev Med Chile 2011; 139: 60-65).

Key words: Community health services: Pregnancy in adolescence; Social support.
$\mathrm{E}$ l embarazo y la maternidad adolescente son considerados un problema social por las consecuencias biopsicosociales que conllevan, tanto para la madre como para sus hijos/as ${ }^{1-3}$.

Entre las estrategias de intervención utilizadas para abordar esta problemática, el foco se ha centrado en la promoción de salud de los niños/as mediante el trabajo con los padres, fortaleciendo las conductas parentales y las condiciones ambientales que los rodean, de manera de prevenir problemas de salud para ambos ${ }^{4}$. Una de estas modalidades de intervención es la visita domiciliaria (VD). Ésta otorga apoyo a la familia y/o individuo en su propio hogar, a través de encuentros habituales realizados por un agente, quien puede ser un profesional o no-profesional ${ }^{5-7}$.
La VD se considera óptima para poblaciones en condiciones de pobreza, ya que son justamente los individuos más vulnerables, los que tienen mayores dificultades para acceder a los sistemas de salud ${ }^{8,9}$, como es el caso de las adolescentes, quienes suelen desconocer las ofertas de salud disponibles ${ }^{10}$.

Estudios enfocados en la parentalidad y estimulación de niños/as, señalan que la VD favorecería el desarrollo de habilidades parentales y la calidad del ambiente familiar, mejoraría el bienestar de la madre, así como el impacto en la salud física y mental del niño/ $\mathrm{a}^{11,12}$.

Actualmente existen divergencias en relación a cuáles son las características apropiadas de los agentes visitadores. La literatura señala que, de- 
pendiendo de los objetivos de la intervención, se necesitarían perfiles distintos. La discusión se ha centrado en la inclusión de visitadores profesionales versus no-profesionales y en su entrenamiento, experiencia, habilidades, capacitación y supervisión ${ }^{4,5,11,13}$. La evidencia disponible muestra que el impacto encontrado depende de las características del visitador, encontrándose fortalezas y limitaciones en cada uno de ellos ${ }^{12}$.

En Chile, existen dos investigaciones que han evaluado la efectividad y/o costo-efectividad de programas de VD, con madres adolescentes, y las visitas han sido realizadas por no profesionales de salud, utilizando distintos diseños metodológicos. Los estudios reportan resultados exitosos en promedio hasta los 15 meses de los niños/as ${ }^{14,15} y$ mejores indicadores de salud mental y nutrición materna $^{15}$.

De esta manera, y conociendo los beneficios de las VD realizadas por agentes no-profesionales de la comunidad, el objetivo de este estudio fue comparar la salud mental materna, el apoyo psicosocial percibido, la asistencia al colegio y el desarrollo infantil en dos cohortes de díadas que han recibido diferentes programas de intervención para madres adolescentes y sus hijos/as a los 12-15 meses. Para tal efecto se compararon aquellas díadas que participaron en el programa "Ayudando a Crecer" de la Vicaría Pastoral Social y de los Trabajadores (en adelante Programa A) y el programa La Pintana-UC realizado en el marco de los proyectos FONDECYT No 1030476 y N 1070836 (en adelante Programa B), respecto a un grupo que no recibió VD. Estos programas se basan en la creencia de que la propia comunidad puede promover cambios en su contexto ${ }^{16}$.

La hipótesis planteada en este estudio es que habría una asociación entre haber recibido algún programa de VD (Programa A o B) con salud mental de la madre, apoyo social percibido, asistencia al colegio y desarrollo infantil, todos evaluados a los 12-15 meses de edad de los hijos/as.

\section{Material y Método}

Se realizó un estudio de corte transversal anidado en dos estudios experimentales (proyectos FONDECYT No1030476 y N¹070836).

Los participantes de este estudio fueron 132 díadas madre-hijo/a. De las díadas, 41 pertenecían al programa A, 46 al programa B, y 45 al grupo control. Entre los criterios de inclusión para los tres grupos se consideró que las madres fueran primíparas y que tuvieran entre 14 y 20 años al momento de la concepción. Todas las díadas se atendían regularmente en el sector público de salud.

La Tabla 1 muestra que los tres grupos de madres que conforman este estudio, son similares

Tabla 1. Características generales de las díadas en estudio por Programa

\begin{tabular}{|c|c|c|c|c|}
\hline & & La Pintana-UC & "Ayudando a Crecer" & Control \\
\hline Número de madres & $N$ & 46 & 41 & 45 \\
\hline \multirow[t]{3}{*}{ Edad materna (años) } & Promedio & 18,42 & 18,05 & 18,51 \\
\hline & Desviación Estándar & 1,54 & 1,69 & 1,39 \\
\hline & Rango & 15 a 21 & 14 a 21 & 16 a 21 \\
\hline \multirow[t]{3}{*}{ Escolaridad materna (años) } & Promedio & 9,9 & 10,03 & 10,23 \\
\hline & Desviación Estándar & 1,39 & 1,98 & 1,76 \\
\hline & Rango & 7 a 12 & 6 a 12 & 5 a 12 \\
\hline Número de hijos & $n$ & 43 & 38 & 41 \\
\hline \multirow[t]{3}{*}{ Edad de hijos (meses) } & Promedio & 12,82 & 12,57 & 13,1 \\
\hline & Desviación Estándar & 0,94 & 0,81 & 0,94 \\
\hline & Rango & 12 a 16 & 12 a 15 & 11 a 15 \\
\hline Sexo de los hijos & $\%$ de hombres & 61 & 45 & 60 \\
\hline
\end{tabular}


en promedio de edad y escolaridad. Respecto a los niños/as, las muestras son equivalentes en edad (promedio de 13,3 meses; rango entre 11,9 y 15 meses) y en la distribución por sexo.

Las díadas madre-hijo/a del programa B, así como del grupo control fueron reclutadas durante el período de un año a través de dos centros de salud ubicados en la comuna de La Pintana, en la Región Metropolitana. La asignación al grupo experimental o al control fue de carácter aleatorio. La información que se presenta sobre estos dos grupos, corresponde a aquellas díadas que terminaron el estudio experimental a los 12/15 meses. Este estudio contó con una pérdida muestral de $12,5 \%$.

Las díadas del programa A se contactaron mediante la Vicaría Pastoral Social y de los Trabajadores, de la Región Metropolitana, y pertenecían a comunas de bajos ingresos.

Las mediciones efectuadas fueron:

Salud Mental Materna: Se evaluó mediante la versión abreviada del cuestionario de Goldberg, estimándose que a mayor puntaje hay mayor riesgo en salud mental. Este instrumento tiene por objetivo la detección de desórdenes mentales de origen neurótico y algunos trastornos de personalidad y desórdenes psicofisiológicos, y excluye aquellos de origen psicótico y orgánico-cerebral ${ }^{17-19}$.

Apoyo Social Percibido: La escala fue tomada del cuestionario "Cómo es tu Familia"20. Consta de 5 ítems, los cuales fueron aplicados a las madres a través de un cuestionario. La confiabilidad interna en esta muestra fue de 0,51 (Alfa de Cronbach).

Satisfacción con la vida: La escala de satisfacción fue tomada del cuestionario "Cómo es tu Familia”. Consta de 9 ítems. La confiabilidad interna en esta muestra fue de 0,73 (Alfa de Cronbach).

Asistencia al colegio: Fue medida a través de la incorporación de la madre al sistema educacional después del parto.

Desarrollo psicomotor infantil: Se midió a través de la Escala de Evaluación del Desarrollo Psicomotor de 0 a 24 meses $^{21}$. La prueba permite obtener un puntaje global del desarrollo y por áreas. De este modo permite distinguir tres grupos de acuerdo al puntaje obtenido y su comparación con baremos de acuerdo a su edad: normales, con riesgo y con retraso. En este estudio se analizó el comportamiento global y en las áreas de lenguaje y social. La prueba fue aplicada en el caso del programa B y control por enfermeras de los Centros de Salud de la comuna, mientras que en el caso del programa A se realizó por estudiantes de enfermería del último año, de la Universidad Católica, supervisadas por una enfermera o pediatra docente. Los resultados de cada evaluación de los niños/as les fueron entregados a la madre y a las coordinadoras del programa.

Maltrato y Negligencia: Se indagó en las fichas clínicas el diagnóstico de maltrato o negligencia; sin embargo, la calidad de este registro no permitió incorporarlo como variable de estudio.

Las tres cohortes de díadas madre-hijo/a evaluadas recibieron intervenciones diferentes. Todas recibieron la atención rutinaria de los programas tradicionales realizados en atención primaria, antes de la implementación del programa Chile Crece Contigo ${ }^{22}$.

La cohorte del programa B formó parte del proyecto FONDECYT No 1030476 y recibió VD mensuales, ejecutadas por monitoras de la comunidad asociadas a los Centros de Salud comunales y capacitadas por las matronas del Centro de Salud, apoyadas por el equipo de investigación (ver detalle, 15). Para estos efectos se diseñó un manual para las monitoras y un conjunto de cartillas para el trabajo en terreno con las adolescentes. Este manual comprende los siguientes tópicos (a) etapa de vida de la joven; (b) salud mental de la joven (identidad, autoestima, proyecto de vida); (c) salud física de la joven; (d) salud física del niño/a y (e) redes de apoyo social. Durante la visita, la monitora definió con la madre los tópicos a tratar. Adicionalmente se le entregó a cada madre un diario para que escribieran lo que les sucedía, sus preguntas y dudas, las que podían ser o no ser comentadas con la monitora en el transcurso de la visita siguiente. El promedio de visitas domiciliarias realizadas fue de 12 al año.

La cohorte del programa A, estuvo a cargo de monitoras voluntarias de la comunidad vinculadas a la pastoral social de sus parroquias $y$ coordinadas por un equipo de acompañamiento de la Vicaría Pastoral Social y de los Trabajadores, de Santiago. El programa se articuló en torno a tres compo- 
nentes: VD, talleres formativos dirigidos hacia las adolescentes y acciones comunitarias (como por ejemplo, coordinación con el colegio de la madre), siendo la primera de ellas el eje principal. Las VD se realizaron de manera periódica de acuerdo a las necesidades establecidas en conjunto con la madre desde el embarazo hasta los 2 años de vida del niño/a. Las visitas correspondieron a un espacio de encuentro y de resolución de dudas respecto del embarazo y la crianza del niño/a, y la posibilidad de integración a redes comunitarias. Como material de apoyo, la Vicaría cuenta con cartillas dirigidas hacia el proceso de crianza en los primeros dos años de vida del hijo/a. Este programa en promedio ejecuta 6 visitas anuales.

La cohorte control correspondió a díadas de dos Centros de Salud de la comuna de La Pintana. Este grupo no recibió VD durante el embarazo y el primer año de vida.

Dada las características del diseño (transversal), las mediciones fueron realizadas a los 12-15 meses de vida de los hijos/as de las tres cohortes. Las mediciones a las madres se realizaron por encuestas que contenían tanto variables sociodemográficas como los instrumentos indicados. Estos fueron administrados por las monitoras comunitarias en los tres grupos de estudio, previo pilotaje. En el caso de los niños/as, las evaluaciones fueron realizadas por profesionales o estudiantes de enfermería de los últimos años.

Para el análisis estadístico se utilizó el software SPSS 15.0. Se analizaron descriptivamente el conjunto y cada grupo, y se realizaron pruebas de comparación paramétricas y no paramétricas entre grupos independientes.

Este estudio contó con la aprobación del Comité de Ética de Investigación de la Escuela de
Psicología de la Universidad Católica de Chile y del Fondo Nacional de Ciencias y Tecnología. Todas las participantes debieron firmar una carta de consentimiento informado para ingresar al estudio.

\section{Resultados}

La salud mental de las madres, determinada por el test de Goldberg, fue diferente entre los grupos $(F(2,128)=3,97, \mathrm{p}=0,02)$. Al realizar contrastes específicos entre grupos, las adolescentes del programa B presentaron mejor salud mental que el grupo control $(t(128)=2,23, \mathrm{p}=0,014)$. No obstante, no hubo diferencias al comparar el nivel de salud mental entre las jóvenes que pertenecen al programa A y aquellas del grupo control $(t(128)=$ $-0,42, \mathrm{p}=0,34)$.

El 25\% del total de las adolescentes evaluadas no se incorporaron al sistema educacional después del embarazo. Al comparar los tres grupos, se encontró que una mayor proporción de adolescentes del programa A siguieron estudiando (41,5\%) en comparación con el grupo control $(15,6 \%)$ y con el grupo B $(19,6 \%)\left(\chi^{2}(1,86)=8,50, p=0,00\right)$.

No se encontraron diferencias significativas entre los tres grupos con respecto al apoyo social percibido por las jóvenes $(F(2,129)=1,512$, p $=$ 0,224); del mismo modo no hubo diferencias en el nivel de satisfacción con diferentes aspectos de la vida $(F(2,129)=0,813, \mathrm{p}=0,446)$.

Con relación a las variables de los niños/as estudiados se encontró que de los 132 evaluados el 3,2\% presentaba riesgo y $96,8 \%$ era normal; no hubo casos con retraso. El desarrollo del lenguaje y social fue normal en el 97\% y 91\% de los niños/ as respectivamente.

Tabla 2. Comparación del desarrollo global, del lenguaje y social, de hijos/as de madres adolescentes

\begin{tabular}{|llcccc|}
\hline Medición & & $\begin{array}{c}\text { La Pintana- } \\
\text { PUC } \\
\text { (n= 41) }\end{array}$ & $\begin{array}{c}\text { Control } \\
\text { (n= 38) }\end{array}$ & $\begin{array}{c}\text { “Ayudando } \\
\text { a Crecer"” } \\
\text { (n= 44) }\end{array}$ & $\begin{array}{c}\text { Total } \\
\text { (n = 123) }\end{array}$ \\
\hline Coeficiente de desarrollo global & Media & 1,00 & 1,03 & 1,01 & 1,01 \\
& DE & 0,07 & 0,10 & 0,12 & 0,10 \\
Rango & $0,83-1,16$ & $0,85-1,22$ & $0,81-1,35$ & $0,81-1,35$ \\
\hline Desarrollo normal & \% del total & 97,56 & 97,36 & 95,45 & 96,75 \\
\hline Desarrollo normal del lenguaje & \% del total & 95,12 & 100,00 & 95,46 & 96,70 \\
Desarrollo normal del área social & \% del total & 90,24 & 100,00 & 86,36 & 91,87 \\
\hline
\end{tabular}


Al comparar los grupos, no se encontraron diferencias significativas en el coeficiente global de desarrollo de los niños/as a los 12-15 meses $(F$ $(2,102,3)=0,764, \mathrm{p}=0,469$; test de ANOVA con corrección Brown-Forsythe). No se encontraron diferencias en las frecuencias de riesgo entre grupos.

Se observó una tendencia no significativa para un mejor desempeño en lenguaje en el grupo B respecto de controles y del programa A (Tabla 2). No se encontraron diferencias entre grupos con relación a retraso de desarrollo social, sin embargo, se observó una tendencia a una menor proporción en el programa A respecto de los controles $(\mathrm{p}=$ $0,053)$. Esto se ratificó con el mejor desempeño en la prueba para los niños/as de este programa en comparación con el grupo control $(\mathrm{p}=0,01)$; sin embargo, no hay diferencias con el grupo A (Tabla 2).

\section{Discusión}

Los programas de VD estudiados muestran que existen resultados positivos fundamentalmente para la madre adolescente.

El programa B, que se caracterizó por ser altamente estandarizado, con visitas mensuales durante el embarazo y el primer año de vida (promedio 12 visitas anuales), utilizando un manual y cartillas de apoyo con contenidos específicos, mostró resultados favorables fundamentalmente en la salud mental de la adolescente. Mientras que, el programa $\mathrm{A}$, realizado de manera menos estandarizada con visitas domiciliarias de número variable (promedio de 6 visitas anuales) y con contenidos variables, pero con un fuerte énfasis en la red social, mostró impacto en la permanencia y reincorporación al ámbito escolar.

En los hijos/as los resultados no muestran diferencias estadísticamente significativas en las frecuencias de riesgo y retraso global, del lenguaje y social. Sin embargo, se observa una tendencia (no significativa) a un mejor desempeño, en lenguaje para el caso del programa B y social en el caso de A.

Estos resultados no sorprenden, dado que las evidencias internacionales muestran que el mayor efecto observado de las visitas realizadas por noprofesionales es en las variables asociadas a los padres/madres ${ }^{11}$. Por el contrario, suele encontrarse un efecto marginal en los hijos/as, destacándose en la investigación el papel de los profesionales de la salud en este tema ${ }^{23}$.

Es importante destacar que, dado que fue un estudio transversal, el resultado obtenido no es posible atribuirlo directamente a los Programas. Sin embargo, en el proyecto FONDECYT No 1030476, en un modelo experimental que comparó la cohorte control con la cohorte B, se observaron efectos significativos en salud mental de la madre ${ }^{15}$.

Pese a que los contenidos de las visitas de ambos programas revelan la importancia de la crianza de los hijos/as, es posible que la falta de diferencias observadas entre los grupos se deba a que en el desarrollo infantil temprano (menores de 1 año) incidan otras variables que no se midieron en este estudio. También es posible que haya una baja potencia del estudio, dado el tamaño muestral obtenido.

Se puede entonces, plantear la hipótesis que las VD realizadas por no-profesionales tienen un impacto directo sobre las adolescentes, según el contenido preferente desarrollado en cada modelo (más salud mental versus más redes). Esto nos plantea la pregunta de cuáles son los mecanismos indirectos más adecuados para impactar en el desarrollo de los niños/as ${ }^{11,12}$.

Estos resultados muestran la importancia de implementar las VD como estrategia de intervención para mejorar el bienestar biopsicosocial de las madres y sus hijos/as en un contexto de deprivación. Por otra parte, muestra la relevancia de considerar recursos comunitarios (no profesionales) como un valor potencial en países que cuentan con menos recursos profesionales para la atención del niño/a y su familia. Estudios experimentales con diversas modalidades contribuirán a verificar las hipótesis propuestas y proponer los modelos de visita más adecuados a nuestro contexto.

Agradecimientos: $\mathrm{Al}$ equipo de los Centros de Salud El Roble y Santo Tomás de la comuna de La Pintana, en particular a Francis Ciampi y Fabiola Olcay; también al equipo de la Vicaría Pastoral Social y de los Trabajadores, en particular a Maribel Gálvez, Marisol Panatt y Gladys Araya.

\section{Referencias}

1. Montenegro H. Educación sexual de niños y adolescentes. Rev Med Chile 2000; 128: 571-3. 
2. Spivak H, Weitzman M. Social barriers faced by adolescents and their Children. JAMA 1987; 11: 28-34.

3. Manlove J. Early motherhood in an intergenerational Perspective: The Experience of a British Cohort”. J Marriage and the Family 1997; 59: 132-46.

4. Olds D. Prenatal and infancy home visiting by nurses: From Randomized trials to community replication. Prevent Science 2002; 3: 153-72.

5. Hiatt S, Sampson D, Baird D. Paraprofessional home visitation: Conceptual and pragmatic considerations. J Comm Psychol 1997; 25: 77-93.

6. Olds D, Korfmacher J. The evolution of a program of research on prenatal and early childhood home visitation. J Comm Psychol 1997; 25: 1-9.

7. Duggan A, Caldera D, Rodríguez K, Burell L, Rohde C, Crowne $\mathrm{S}$. Impact of a state home visiting program to prevent child abuse. Child Abuse \& Neglect 2000; 31: 801-27.

8. Gomby DS, Culross PL, Behrman RE. Home visiting: recent program evaluation-analysis and recommendations. The Future of Children 1999; 9: 4-26

9. Dieterich S, Smith K, Swank P, Hebert H. Impact of community mentors on maternal behaviors and child outcomes. J Early Intervent 2006; 28: 111-24.

10. Jacobson L, Richardson G, Parry-Langdon N, Donvan C. How do teenagers and primary healthcare providers view each other? A overview of key themes. Br J Gen Pract 2001; 51: 811-6.

11. Elkan R, Kendrick D, Hewitt M, Robinson JJA, Tolley $\mathrm{K}$, Blair $\mathrm{M}$, et al. The effectiveness of domiciliary health visiting: A systematic review of international studies and selective review of the British literature. Health Technol Assess 2000; 4: 251-5.

12. Sweet MA, Appelbaum MI. Is home visiting an effective strategy? A meta-analytic review of home visiting programs for families with young children. Child Develop 2004; 74: 1435-56.
13. Olds D, Robinson J, O’Brien R, Luckey DW, Pettitt LM, Henderson CR Jr, et al. Home visiting by paraprofessionals and by nurses: A randomized, Controlled Trial. Pediatrics 2002, 110: 486-96.

14. Cumsille P, Ramírez V. Evaluación de un programa comunitario destinado a favorecer el desarrollo psicosocial de madres adolescentes y sus hijos. Psyhke 1999; 8: 17-30.

15. Aracena M, Krause M, Pérez JC, Méndez MJ, Soto M, Pantoja, T, et al. A cost-effectiveness evaluation of a home visit program for adolescent Mothers. J Health Psychol 2009; 14: 878-87.

16. Mendive S. Entrevista al Doctor Juan Marconi, Creador de la psiquiatría intracomunitaria. Reflexiones acerca de su legado para la psicología comunitaria Chilena. Psyke 2004; 13: 187-99.

17. Goldberg D. The detection of psychiatric illness by questionaire. New York, Oxford University Press, 1972.

18. Trucco M, Campusano M, Larraín S. Un cuestionario para detectar desórdenes emocionales: estudio de validación preliminar. Rev Chil Neuropsiquiat 1979; 17: 20-5.

19. Araya R, Wynn R, Lewis G. Comparison of two self administered psychiatric questionnaires (GHQ-12 and SRQ-20) in primary care in Chile. Social Psychiat Psychiatric Epidemiol 1992; 27: 168-73.

20. Hidalgo CG, Carrasco E. (1999). Salud Familiar: Un modelo de atención integral en la atención primaria. Santiago, Ediciones Universidad Católica de Chile, 1999.

21. Rodríguez S, Arancibia V, Undurraga C. Escala de evaluación del desarrollo psicomotor 0-24 meses. Santiago, Editorial Cronopios, 2008.

22. Ministerio de Salud, Chile Crece Contigo. Orientaciones técnicas. Visita domiciliaria integral para el desarrollo biopsicosocial de la infancia. Santiago: Gobierno de Chile, 2008.

23. Olds D. Prenatal and infancy home visiting by nurses: from randomized trials to community replication. Prevent Sci 2002; 3: 153-72. 